\title{
Application of artificial neural networks to forecasting water quality in a chloraminated water distribution system
}

\author{
$\underline{\text { Wenyan Wu }}^{\text {a }}$, Graeme C. Dandy ${ }^{\mathrm{a}}$ and Holger R. Maier ${ }^{\mathrm{a}}$ \\ ${ }^{a}$ School of Civil, Environmental and Mining Engineering, University of Adelaide \\ Email: wwu@civeng.adelaide.edu.au
}

\begin{abstract}
Accurate forecasting of disinfection residuals in a water distribution system (WDS) is essential for optimal control of disinfectant dosing in order to maintain good water quality within the system. The most commonly used disinfectant in the drinking water treatment process throughout the world is chlorine. Previously, artificial neural networks (ANNs) have been used successfully to predict chlorine residuals in chlorinated WDSs. Recently, an increasing number of water utilities are using chloramine as a secondary disinfectant in WDSs. Chloramine has several advantages compared with chlorine including fewer issues with disinfection by-products (DBP) and longer persistence. However, limited research has been conducted into the modelling of disinfection residuals (chlorine and free ammonia) in a chloraminated WDS. In this research, a general regression neural network (GRNN) has been used to forecast chlorine and free ammonia residuals in one section of the Goldfield and Agricultural Water System (G\&AWS) east of Perth.
\end{abstract}

In this study, the system under investigation spans from the original chlorine and ammonia dosing location at Mundaring pump stations to Goomalling pump station. Ten water quality variables and six flow variables have been used for the development of both chlorine and free ammonia forecasting models. The data were collected at 10 minutes intervals over a one-year period from February 2009 to January 2010. They have been converted into hourly data for this study. A maximum of 94 lags are used, which result in a total of 1,536 potential inputs for each model. In order to select appropriate inputs for each of the models within a reasonable time, a three-step input selection procedure using both mutual information (MI) and partial mutual information (PMI) has been used, which results in 12 selected inputs for the chlorine forecasting model and 10 selected inputs for the free ammonia forecasting model. A deterministic data splitting method called DUPLEX has been used to divide the data into the training (60\%), testing (20\%) and validation (20\%) sets.

The modelling results indicate that ANNs have the ability to forecast chlorine residuals in a chloraminated WDS. This provides a useful tool that can be used to assist in optimal control of disinfectant levels in WDSs. However, further information on both the input variables and the hydraulics of the system is required in order to improve the performance of the chlorine forecasting model. In contrast, the free ammonia forecasting model performed poorly and cannot be used to provide accurate forecasts of free ammonia levels in the system. This is mainly because the free ammonia data collected were estimated based on the difference between the total ammonia nitrogen and the monochloramine nitrogen, and therefore are not accurate. As a result, accurate free ammonia analysers are required in order to obtain precise free ammonia data for the development of an accurate ANN model for the forecasting of the free ammonia levels in a WDS.

Keywords: Artificial neural networks (ANNs), general regression neural networks (GRNNs), water quality, chloramine, water distribution systems (WDSs) 
Wu et al., Application of artificial neural networks to forecasting water quality...

\section{INTRODUCTION}

A critical goal of all water utilities is to provide safe drinking water to consumers. This goal is often achieved (in part) by using disinfectants to remove harmful micro-organisms contained in drinking water. The most commonly used disinfectant in drinking water treatment process throughout the world is chlorine (Rodriguez and Sérodes, 1999). Recently, an increasing number of water utilities around the world are using chloramine instead of chlorine as a secondary disinfectant in WDSs for maintaining water quality due to the advantages of chloramine over chlorine (United States Environmental Protection Agency, 2011). Compared to chlorine, the reaction of chloramines with organic matter is slower and as a result, little or no regulated disinfection by-products (DBPs), such as trihalomethanes (THM), are formed in chloraminated systems. Monochloramine is more chemically stable than chlorine, which makes it remain active within WDSs much longer. Therefore, chloramines are ideal secondary disinfectants for long WDSs. In addition, chloramines do not have any taste or odour.

There are also some drawbacks of using chloramines as a disinfectant for drinking water (Lenntech Water treatment \& purification Holding B.V, 2009). Unlike chlorine, chloramines do not rapidly dissipate on standing or by boiling and need to be removed by using special techniques, such as granular activated carbon filtration systems. The main concern over chloraminated drinking water systems is nitrification. High free ammonia levels in a chloraminated system can serve as nutrients for nitrifying bacteria in the water, which can cause nitrate levels in the water to increase. Nitrate is converted to nitrite in the stomach, which causes the oxygen level in the blood to fall. Nitrification in drinking water can cause potential health problems, especially for babies under six months old (Blue Baby Syndrome). Ammonia can also cause corrosion of lead and copper, which can lead to increased lead concentrations in drinking water. Therefore, there is a need to model not only chlorine residuals but also free ammonia residuals in chloraminated drinking water systems.

Traditionally, water quality in a chlorinated WDS has been predicted using process-based models, which often assume chlorine decay follows a first order reaction (Gibbs et al., 2006). However, this requires a good understanding of the system and extensive, accurate hydraulic data. Alternatively, data-driven statistical models, such as artificial neural networks (ANNs), can be used to determine disinfectant residual levels in a WDS based on empirical relationships between a number of system variables (Gibbs et al., 2006). The advantages of data-driven statistical models over process-based models in water quality prediction are that explicit knowledge of the actual chemical processes in the system is not required. This feature of data-driven statistical models is extremely useful for disinfection control, where the estimation of process-based model parameters is imprecise or difficult to achieve (Rodriguez et al., 1997) or the data required for developing a process-based model are not available.

ANNs have already been successfully applied to model chlorine concentrations in WDSs. Rodriguez et al. (1997) used an ANN model to simulate chlorine residuals in a Severn Trent Water Ltd (U.K.) distribution system and found that ANNs have a promising capacity for reproducing the dynamics of chlorine decay within a WDS. Rodriguez and Sérodes (1999) compared a linear autoregressive model with a non-linear ANN model for predicting chlorine residuals in two Canadian drinking water systems. The results demonstrate that ANN models have the ability to detect non-linear complex relationships between data and that, for the case studies considered, the ANN model gives better predictions than the linear autoregressive model for some water treatment conditions. Gibbs et al. (2006) applied three data-driven techniques (including two ANN models) to predict chlorine residuals at two key locations in the Hope Valley WDS (north of Adelaide, South Australia). The results demonstrated the ability of ANNs to predict chlorine concentrations in a complex WDS for which a hydraulic model is not available and only sparse, routinely measured data are available to be used as inputs. Bowden et al. (2006) successfully applied an ANN model to forecast chlorine concentrations in the Myponga WDS, south of Adelaide. The results demonstrated that ANN models are able to forecast chlorine concentrations in a WDS to a high level of accuracy and for up to 72 hours in advance. More recently, May et al. (2008) developed an ANN model using an improved nonlinear input variable selection (IVS) algorithm for forecasting chlorine residuals in a WDS and compared it with an existing approach. The ANN model with the improved IVS algorithm was found to provide accurate predictions with significantly fewer inputs.

To date, little research has been carried out on forecasting disinfection residuals in chloraminated WDSs. This is partially because chloramine decay in a WDS with organic matter has yet to be fully understood due to the complex series of chemical reactions involved (Duirk et al., 2005). For the same reason, it is difficult to forecast water quality in chloraminated WDSs using a process-based model. For example, in a study by Alexander and Boccelli (2010) the authors found that a multi-species water quality process model (EPANETMSX) cannot characterise the changes of the whole range of species throughout chloraminated WDSs. Therefore, in this paper, it is proposed to develop a data-driven ANN model to forecast water quality in a 
Wu et al., Application of artificial neural networks to forecasting water quality...

chloraminated WDS - the Goldfield and Agricultural Water System (G\&AWS) east of Perth. This paper presents a preliminary study for Water Quality Research Australia project 1032-10. In a later stage of the project, it is anticipated that automatic optimal control of chlorine and ammonia dosing at a water treatment plant (WTP) will be developed to maintain water quality in the chloraminated WDS and minimise potential risks associated with chloramine disinfection.

\section{SYSTEM DESCRIPTION}

The system investigated in this study is part of the Goldfield and Agricultural Water System (G\&AWS) in Western Australia, as shown in Figure 1. The G\&AWS runs for $600 \mathrm{~km}$ from Mundaring weir (east of Perth) to the goldfields at Kalgoorlie. The G\&AWS was built more than 100 years ago from 1898 to 1903 and is still considered as one of the greatest hydraulic engineering works in the world. The original pipeline was built by a team lead by Western Australia's then Engineer-in-Chief C.Y. O'Connor. The original pipeline was 552 kilometres long, consisting of eight pump stations and two reservoirs at Cunderdin and Bullabulling, respectively.

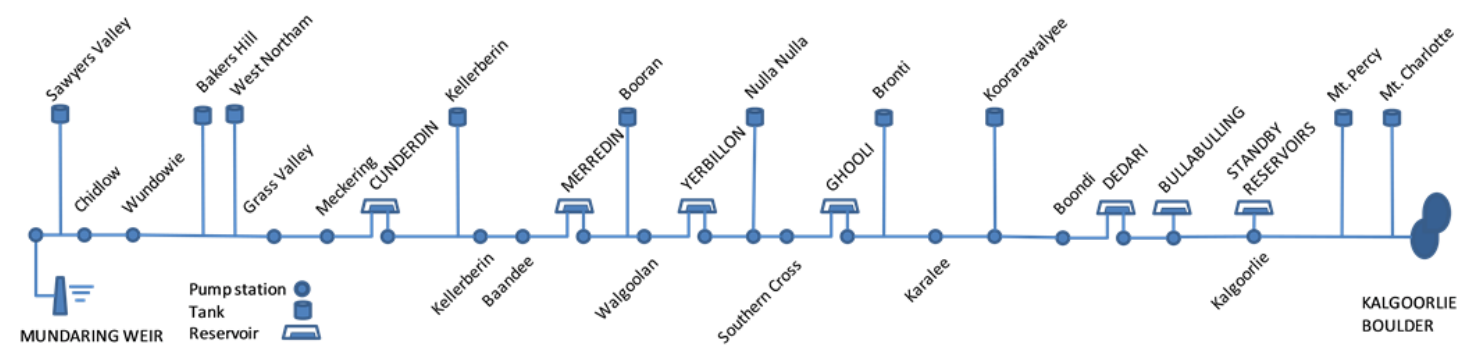

Figure 1. A schematic of the G\&AWS (modified from Water Corporation (2005))

Over the years, extensive alterations and enlargements have been made to the pipeline: the number of pump stations has increased from 8 to 20 and the number of reservoirs from two to seven; an additional nine storage tanks have been constructed along the main pipeline; and a number of extension systems have also been built from the main pipeline. The capacity of the pipeline to supply water to the eastern goldfields has increased by about 8,000 megalitres per year since the pipeline first delivered water to Coolgardie and Kalgoorlie-Boulder (Water Corporation, 2005). In the 2009-2010 financial year, 28,546 megalitres of water were pumped from the Mundaring Reservoir, of which 26,658 megalitres were exported to the G\&AWS.

The system being modelled in this study consists of the trunk main from the initial chlorine and ammonia dosing point at the Mundaring pump stations to Grass Valley and part of the "BP" Extension from Grass Valley to Goomalling pump station, which is about one kilometre downstream of the Goomalling Tanks (Figure 2). There are two major offtake pipelines along the main pipeline at Northam (the "BH" and "BG" extensions). The system is about 150 kilometres long, which provides a suitable reaction time for modelling the chloramines.

Figure 2. A schematic of the modelled system (Goomalling tanks are in the dashed oval)

\section{ANN MODEL DEVELOPMENT}

Maier et al. (2010) reviewed the methods used to develop ANN models for the prediction of water resource variables in river systems. In the paper, their authors divided the ANN model development process into the following seven steps: 1) choice of potential inputs and outputs; 2) data processing; 3) input selection; 4) data division; 5) selection of model architecture; 6) optimisation of model parameters; and 7) model validation. In this study, the chlorine and free ammonia forecasting models are developed in accordance with these steps.

\subsection{Choice of inputs and outputs and data processing}

The choice of inputs is determined by the data available. In this study, 10 water quality variables (ammonia, chlorine and $\mathrm{pH}$ at Mundaring pump stations $\mathrm{A}$ and $\mathrm{B}$, chlorine at Sawyers Valley tanks, previous values of chlorine, free ammonia and water temperature at Goomalling pump station) and six flow variables (flow at 
Wu et al., Application of artificial neural networks to forecasting water quality...

Sawyers Valley tanks, inlet, outlet, and inlet reserve flows at Northam, and flows to the North and the East at Grass Valley tanks) are used. It should be noted that the free ammonia concentrations at Goomalling pump station are estimated based on the difference between the total ammonia nitrogen and monochloramine nitrogen concentrations, which may contain noise due to the existence of other chemicals containing nitrogen (such as dichloramine and nitrate) in the system. The outputs are concentrations of chlorine and free ammonia at Goomalling pump station at time $t$, which are predicted using two separate models in this study. The data have been collected at 10-minute intervals for a one-year period from 6am $2^{\text {nd }}$ February 2009 to $11 \mathrm{pm} 31^{\text {st }}$ January 2010. Data between $11 \mathrm{am} 25^{\text {th }}$ June 2009 and $1 \mathrm{pm} 29^{\text {th }}$ June 2009 are not available due to equipment connection problems. Linear interpolation is used to fill in other missing data. In this study, the data collected at 10-minute intervals are converted to average hourly values, which results in a total of 8,439 data records. A maximum lag of 96 (four days) is considered in relation to potential model inputs, which results in 1,536 potential inputs for each of the two forecasting models.

\subsection{Input selection}

Determination of appropriate inputs is an important step in ANN model development, as the inclusion of too few or too many inputs are both undesirable (Maier et al., 2010). Exclusion of important inputs will result in a loss of important information in the model development process, which can lead to a model that cannot fully describe the relationship between the inputs and outputs. Inclusion of too many correlated or extraneous inputs will not only increase the computational time required for model development, but also increase the likelihood of overfitting the data (Maier et al., 2010).

In this study, both mutual information (MI) and partial mutual information (PMI) are used to select the appropriate inputs for the forecasting of chlorine and free ammonia levels in the WDS (May et al., 2008). Due to the large number of potential inputs for both models, a three-step procedure, which is similar to the one used by Bowden et al. (2005), is used to select the final inputs, in order to reduce computational time. First of all, the MI values for all 96 lags of each of the 16 input variables are calculated, so that the significant lags of each input variables can be determined. Secondly, the PMI algorithm is applied to the selected lags of each of the 16 input variables and the Akaike information criterion (AIC) is used to select significant lags of each input variable. In the last step, the PMI algorithm is applied to the significant lags of all input variables selected in the second step. In this step, the AIC stopping criterion resulted in selection of only one input for the chlorine forecasting model and two inputs for the free ammonia forecasting model. In order to obtain a reasonable number of inputs for both models, the 95th percentile critical value for the sample size of 8,439 (May et al., 2006) is used to select the final inputs for both models. As a result, 12 inputs are selected for the chlorine forecasting model and 10 inputs are selected for the free ammonia forecasting model. The final selected inputs for both models are summarised in Table 1. For more details on the PMI based input selection algorithm and the AIC-based criterion, refer to May et al. (2008).

Table 1. Summary of selected inputs

\begin{tabular}{|l|l|l|l|}
\hline \multicolumn{2}{|c|}{ Final inputs for chlorine forecasting model } & \multicolumn{2}{c|}{ Final inputs for free ammonia forecasting model } \\
\hline Input variables & Lags & Input variables & Lags \\
\hline Mundaring pump station A Chlorine & 11,21 & Sawyers Valley Flow & 1 \\
\hline Mundaring pump station B Chlorine & 9 & Sawyers Valley Chlorine & 17 \\
\hline Sawyers Valley Flow & 16 & Grass Valley Flow to North & $7,13,19$ \\
\hline Sawyers Valley Chlorine & 8,15 & Goomalling Free Ammonia & 1,4 \\
\hline Grass Valley Flow to East & 1,9 & Goomalling Temperature & 1,9 \\
\hline Goomalling Temperature & $1,12,13$ & Goomalling Chlorine & 6 \\
\hline Goomalling Chlorine & 1 & & \\
\hline
\end{tabular}

\subsection{Data division}

In the ANN model development process, data are usually divided into three subsets: training, testing and validation sets. The training set is used to train (calibrate) the model, the testing set is used in cross-validation to avoid over-fitting and the validation set is used to test the performance of the trained model. In order to develop the best model and fully test it for the given dataset, the training, testing and validation subsets need to have similar statistical properties (Maier et al., 2010).

In this study, $60 \%$ of the data are used for calibration, $20 \%$ for testing and $20 \%$ for validation. This was achieved using a data splitting method called DUPLEX. DUPLEX was developed by Snee (1977) based on one of the earliest data splitting algorithm called CADEX or Kennard-Stone sampling (Kennard and Stone, 1969). The DUPLEX method used in this study was modified by May et al. (2010), which can generate three 
Wu et al., Application of artificial neural networks to forecasting water quality...

datasets for training, testing and validation. DUPLEX is a deterministic method with no variation. Therefore, it is an ideal benchmark data splitting method. One significant drawback of DUPLEX is its computational complexity, which may prohibit its use on large datasets (May et al., 2010). However, this was not an issue for this case study.

\subsection{Selection of model architecture and optimisation of model parameters}

In this paper, the general regression neural network (GRNN) is used to forecast both chlorine and free ammonia concentrations at the Goomalling pump station. The objective of the models is to map the relationship between a single water quality parameter value (either chlorine or free ammonia concentration) at a downstream location in a WDS and multiple inputs, including the dosing rates of chlorine and ammonia at the initial dosing location at Mundaring pump stations A and B. Therefore, two separate GRNN models are developed in this study: one model for forecasting chlorine concentration; and one model for forecasting free ammonia concentration.

The GRNN, which is classified as a probabilistic neural network (PNN), was first introduced by Specht (1991) and is used in this study because it has been successfully used to model water quality parameters in WDSs (Bowden et al., 2006; May et al., 2008). The architecture of the GRNN consists of four fully connected layers: an input layer, a pattern layer, a summation layer and an output layer. Compared to multilayer perceptrons (MLPs), which have been used more commonly in ANN applications in water resources (Maier et al. 2010), GRNNs are more memory intensive; however, they have a fixed architecture and only one parameter, the bandwidth, that needs to be obtained by calibration. Therefore, a GRNN model is much faster to develop (May et al., 2008). In this study, both of the GRNN models are trained using Brent's method. For more details on the GRNN used in this study, refer to May et al. (2008).

\subsection{Model performance criteria}

A multi-criteria approach is adopted in this study to assess the models developed. Four statistical error and goodness-of-fit measures, including the root mean square error (RMSE), the mean absolute error (MAE), the square of Pearson $r\left(r^{2}\right)$ and the Nash-Sutcliff model efficiency coefficient $(E)$, are used, in order to determine how well the predicted response matches different aspects of the measured response (e.g. peak, average etc.).

\section{RESULTS AND DISCUSSION}

The training, testing and validation results for both the chlorine and the free ammonia forecasting models are summarised in Table 2. The calibrated bandwidth of the GRNN models is 0.4011 for the chlorine forecasting model and 0.6865 for the free ammonia forecasting model. It can be seen from the table that for both models, the training datasets are predicted to a higher level of accuracy compared to the testing and validation datasets. This is evident in the lower forecasting errors and higher Pearson $r^{2}$ and Nash-Sutcliff model efficiency coefficient $E$ values of the training sets. This result is expected, as the models are calibrated mainly based on the information contained in the training datasets. For both models, the RMSEs are much higher than the MAEs for all three datasets, which indicates that both models generate higher than average forecasting errors at some data points. It can also be seen from Table 2 that the generalization ability of the chlorine forecasting model is much better than that of the free ammonia forecasting model. Even though the $r^{2}$ and $E$ values of the testing and validation sets of the chlorine forecasting model are lower than those of the training set, they are still very close to one. However, the $r^{2}$ and $E$ values of the testing and validation sets of the free ammonia forecasting model are much lower than those of the training set and are only around 0.6 .

Table 2. Model performance for the chlorine and the free ammonia forecasting models

\begin{tabular}{|l|c|c|c|c|c|c|c|c|}
\hline & \multicolumn{4}{|c|}{ Chlorine forecasting model } & \multicolumn{4}{c|}{ Free ammonia forecasting model } \\
\hline Bandwidth & \multicolumn{3}{|c|}{0.4011} & \multicolumn{4}{c|}{0.6865} \\
\hline Performance & RMSE & MAE & $r^{2}$ & E & RMSE & MAE & $r^{2}$ & E \\
\hline Training set & 0.025 & 0.016 & 0.996 & 0.996 & 0.070 & 0.043 & 0.877 & 0.874 \\
\hline Testing set & 0.061 & 0.032 & 0.974 & 0.974 & 0.159 & 0.105 & 0.604 & 0.594 \\
\hline Validation set & 0.062 & 0.034 & 0.974 & 0.974 & 0.159 & 0.105 & 0.613 & 0.606 \\
\hline
\end{tabular}

The plots of the one-hour forecasts of chlorine and free ammonia obtained using the validation datasets are shown in Figures 3(a) and 3(b), respectively. As the validation dataset is independent of the training process 
of the model, it provides a better representation of the model's overall performance. It can be seen from Figure 3 that the overall performance of the chlorine forecasting model is satisfactory and, most of the time, is capable of providing an accurate chlorine forecast at the Goomalling pump station. For a few data points (as shown in the circles in Figure 3(a)), the chlorine forecasting model cannot generate accurate forecasts, which is in agreement with the fact that the RMSE is higher than the MAE, as shown in Table 2. While the free ammonia model represents the general trend of the data reasonably well, it does not represent the extremes of ammonia levels observed in the data.
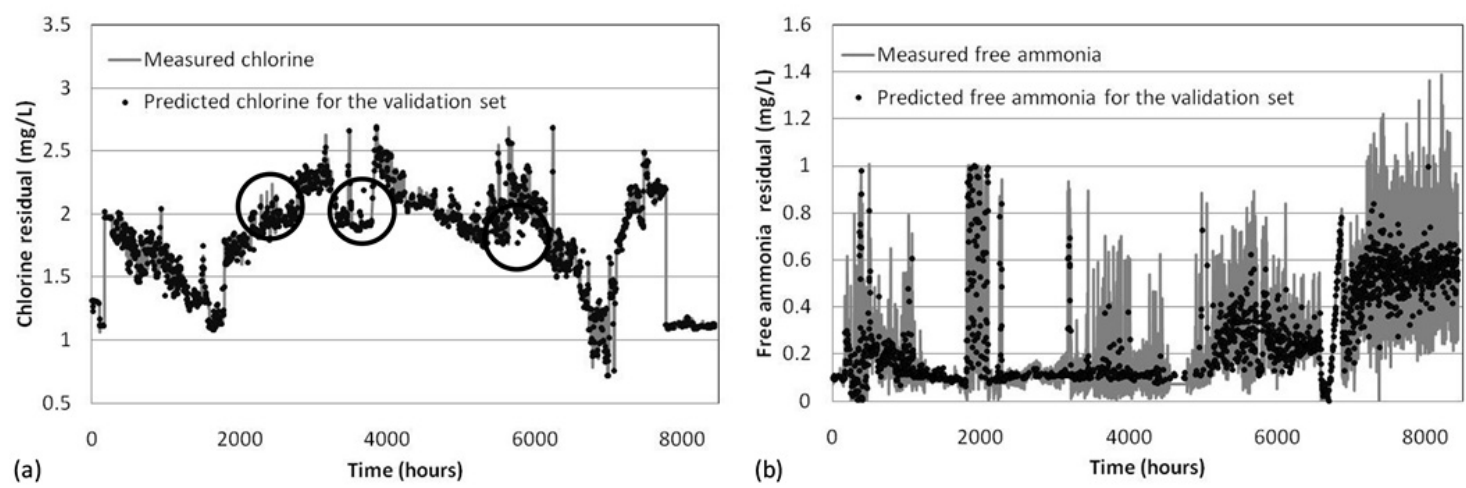

Figure 3. Validation set one-hour forecasts of (a) chlorine and (b) free ammonia

There are a number of reasons for the lack of accurate chlorine forecasts at some of the data points. Firstly, the WDS from the Mundaring pump stations to the Goomalling pump station is very complex, with multiple tanks and offtakes, which may block or interfere with the signals from the initial dosing points at the Mundaring pump stations. This is also a very important reason for the fact that only a few inputs at the Mundaring pump stations are selected for the chlorine forecasting model and none are selected for the free ammonia forecasting model. Secondly, there are two initial chlorine and ammonia dosing points at the Mundaring pump stations (A and B), which are used as separate inputs in this study. However, the total chlorine and ammonia doses from both Mundaring pump stations may be better predictors of the chlorine and free ammonia levels at Goomalling pump station. This point requires further investigation. Thirdly, even though there is only a 4-day period of missing data during the one-year data collection period, there may be erroneous values for some input variables during this period, which were not identified in this study. These erroneous values can distort the mapping of the relationship between the inputs and the outputs, which can result in high forecasting errors at certain data points. As a result, further information on the input variables is required to either correct the erroneous values or remove some input variables from the model development process if they are identified to have erroneous data over long periods.

Apart from the reasons given above, the main reason that the free ammonia forecasting model has poor performance is that the free ammonia concentrations at Goomalling pump station are not directly measured, but estimated based on the difference between the total ammonia nitrogen and monochloramine nitrogen values. Due to the existence of other chemicals containing nitrogen (such as dichloramine and nitrate) in the system, the free ammonia data are not accurate and contain a lot of noise, which can be clearly observed in Figure 3(b). Therefore, accurate free ammonia analysers are required in order to obtain accurate free ammonia data for the development of accurate free ammonia forecasting models for the WDS under investigation.

\section{CONCLUSIONS}

This paper presents a preliminary study for Water Quality Research Australia project 1032-10. This study attempts to map the relationship between a number of water quality and hydraulic input variables and water quality variables at a downstream location (either the chlorine residual or the free ammonia residual) for part of the Goldfield and Agricultural Water Systems (G\&AWS) using general regression neural networks (GRNNs). In total, 10 water quality and six flow variables have been used. A three-step procedure using both the mutual information (MI) and partial mutual information (PMI) have been used to select appropriate inputs for both the chlorine forecasting and free ammonia forecasting models. A deterministic data splitting method called DUPLEX has been used to divide the data into the training, testing and validation sets.

The preliminary results provided in this study show that the GRNN is capable of forecasting chlorine residuals in a chloraminated WDS. However, further information on both the input variables and the 
Wu et al., Application of artificial neural networks to forecasting water quality...

hydraulics of the system is required in order to improve the performance of the chlorine forecasting model. In contrast, similar conclusions cannot be drawn regarding the forecasting ability of the free ammonia forecasting model due to the poor performance of the model. However, it has been identified that the main reason for the bad performance of the free ammonia forecasting model is that the free ammonia data contain too much noise and are not accurate. Therefore, accurate free ammonia analysers are required in order to collect reliable free ammonia data for the development of accurate ANN models to forecast the free ammonia levels in the system.

\section{ACKNOWLEDGMENTS}

This research was funded by Water Quality Research Australia (WQRA) as part of the project 1032-10. The authors would like to thank Mr. Ralph Henderson and Mr. Ian Scott from Water Corporation for their help with this research (data collection).

\section{REFERENCES}

Alexander, M.T. and Boccelli, D.L. (2010). Field verification of an integrated hydraulic and multi-species water quality model. Water Distribution System Analysis 2010. Tucson, AZ, USA.

Bowden, G.J., Maier, H.R. and Dandy, G.C. (2005). Input determination for neural network models in water resources applications. Part 2. Case study: forecasting salinity in a river. Journal of Hydrology 301(1-4): 93-107.

Bowden, G.J., Nixon, J.B., Dandy, G.C., Maier, H.R. and Holmes, M. (2006). Forecasting chlorine residuals in a water distribution system using a general regression neural network. Mathematical and Computer Modelling 44(5-6): 469-484.

Duirk, S.E., Gombert, B., Croué, J.-P. and Valentine, R.L. (2005). Modeling monochloramine loss in the presence of natural organic matter. Water Research 39(14): 3418-3431.

Gibbs, M.S., Morgan, N., Maier, H.R., Dandy, G.C., Nixon, J.B. and Holmes, M. (2006). Investigation into the relationship between chlorine decay and water distribution parameters using data driven methods. Mathematical and Computer Modelling 44(5-6): 485-498.

Kennard, R.W. and Stone, L.A. (1969). Computer Aided Design of Experiments. Technometrics 11(1): 137148.

Lenntech Water treatment \& purification Holding B.V. (2009). Disinfectants Chloramines. Accessed November 2010, http://www.lenntech.com/processes/disinfection/chemical/disinfectantschloramines.htm.

Maier, H.R., Jain, A., Dandy, G.C. and Sudheer, K.P. (2010). Methods used for the development of neural networks for the prediction of water resource variables in river systems: Current status and future directions. Environmental Modelling \& Software 25(8): 891-909.

May, R.J., Dandy, G.C., Maier, H.R. and Fernando, T.M.K. (2006). Critical Values of a Kernel Densitybased Mutual Information Estimator. Proceedings of IJCNN'2006.: 4898 4903

May, R.J., Dandy, G.C., Maier, H.R. and Nixon, J.B. (2008). Application of partial mutual information variable selection to ANN forecasting of water quality in water distribution systems. Environmental Modelling \& Software 23(10-11): 1289-1299.

May, R.J., Maier, H.R. and Dandy, G.C. (2010). Data splitting for artificial neural networks using SOMbased stratified sampling. Neural Networks 23(2): 283-294.

May, R.J., Maier, H.R., Dandy, G.C. and Fernando, T. (2008). Non-linear variable selection for artificial neural networks using partial mutual information. Environmental Modelling \& Software 23(10-11): 1312-1326.

Rodriguez, M.J. and Sérodes, J.-B. (1999). Assessing empirical linear and non-linear modelling of residual chlorine in urban drinking water systems. Environmental Modelling and Software 14(1): 93-102.

Rodriguez, M.J., West, J.R., Powell, J. and Serodes, J.B. (1997). Application of two approaches to model chlorine residuals in Severn Trent Water Ltd (STW) distribution systems. Water Science and Technology 36(5): 317-324.

Snee, R.D. (1977). Validation of regression models: Methods and examples. Technometrics 19(4): 415-428.

Specht, D.F. (1991). A general regression neural network. Neural Networks, IEEE Transactions on 2(6): 568576.

United States Environmental Protection Agency. (2011). Chloramines in drinking water. Accessed February 2011, http://water.epa.gov/lawsregs/rulesregs/sdwa/mdbp/chloramines index.cfm.

Water Corporation. (2005). Securing water for the goldfields. Accessed March 2011, http://www.watercorporation.com.au/ files/PublicationsRegister/12/Goldfields_water.pdf. 